\title{
Leadless pacemaker implantation via azygos vein in a patient with absence of the hepatic segment of the inferior vena cava
}

\author{
Mário Oliveira*, Dinis Mesquita, Pedro S. Cunha, Ana S. Delgado, and Rui C. Ferreira
}

Arrhythmology Unit, Santa Marta Hospital, Rua Santa Marta, 1169-024, Lisbon, Portugal

* Corresponding author. Tel: 351 213594311; fax: 351 213531983. E-mail address: m.martinsoliveira@gmail.com

An 82-year-old woman with atrial fibrillation, in chronic haemodialysis (arteriovenous fistulae in both arms), underwent a transcatheter aortic valve implantation. She had de novo left bundle branch block, without bradyarrhythmia and was discharged. Two weeks later, she had asystole during a dialysis session and was referred to permanent pacemaker. A decision was made to use the Micra transcatheter leadless pacemaker (Medtronic Inc., Minneapolis, MN, USA). A stiff support wire inserted via the right femoral vein showed a tortuous course and inability to advance through the inferior vena cava. A cine-angiogram documented an enlarged azygos vein with a very tortuous path draining to the superior vena cava (Panel A). An extra stiff guidewire was used, but the 27-Fr hydrophilic sheath and dilator failed to track across (Panel B). Using gentle but sustained pushing and pulling on the extra stiff guidewire, combined with transient

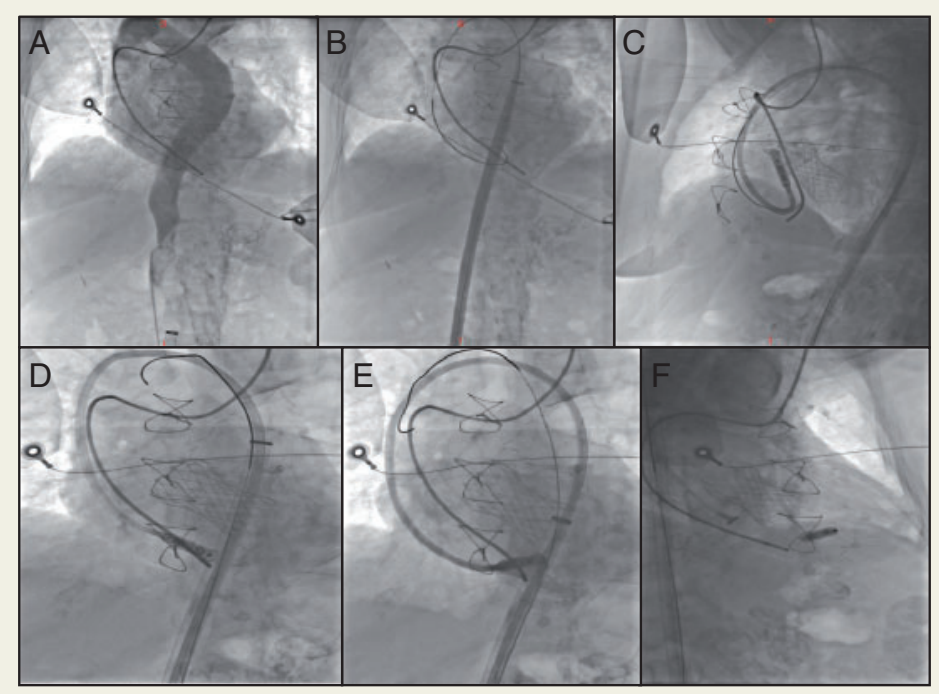
withdraw of the temporary pacing, allowed final advancement of the sheath to the right ventricle (Panels $C$ and $D$ ).

The final position was on the apical septum (Panels $E$ and $F$ ). The pacing threshold at implant was $0.5 \mathrm{~V}$ (at $1 \mathrm{~ms}$ ), the R-wave sensing amplitude was $10.7 \mathrm{mV}$, and the pacing impedance was $680 \Omega$. No procedural complications occurred.

The full-length version of this report can be viewed at: http:/www.escardio.org/Guidelines-\&-Education/E-learning/Clinical-cases/ Electrophysiology/EP-Case-Reports.

Published on behalf of the European Society of Cardiology. All rights reserved. @ The Author(s) 2019. For permissions, please email: journals.permissions@oup.com. 